\title{
Screening of Secondary Hypertension in Private Clinic in Karachi, Pakistan: A Randomised Study
}

\author{
Khalida Soomro $^{1 *}$, Muhammad Ali Soomro
}

\author{
${ }^{1}$ Department of Cardiology, Dow University of Health and \\ Sciences Karachi, Pakistan. \\ ${ }^{2}$ Lecturer in Department of pathology in Bilawal medical college \\ of Liaquat university of Health and Sciences at Jamshoro/ \\ Hyderabad, Pakistan.
}

\author{
*Corresponding author \\ Khalida Soomro, Department of Cardiology,Dow University of Health and \\ Sciences Karachi, Pakistan.
}

Submitted: 13 Oct 2020; Accepted: 03 Nov 2020; Published: 11 Nov 2020

\begin{abstract}
Hypertension has been recognized as a global health concern for developing countries. Secondary hypertension (SH) including endocrine hypertension has been reported to be uncommon and is scarcely described in many of these countries. There is no current nationally representative study. Therefore, we estimated the prevalence of SH among hypertensive patients in private clinic by screening. The goal of the cross-sectional study conducted from May 2017to June2018 was to estimate the prevalence of hypertension in Pakistani population based on data collected from the screening held at private clinic in Karachi. From the initial screening of 566 patients, we suspected 437 (M:187), (F:250) patients to have secondary hypertension and conducted tests for the cause of hypertension, measured plasma aldosterone concentration, plasma renin activity, serum cortisol concentration and plasma catecholamine concentration. As a secondary screening, we referred patients for furosemide plus upright test, captopril renography, dexamethasone suppression test, abdominal ultrasonography (US), abdominal CT. and arteriography investigations. These tests clearly demonstrated that the prevalence of Secondary Hypertension was $8.1 \%$ among 437 patients investigated. 42 were found to have Renal parenchymal disease; 25 were having Acute Renal Failure; 15 patients had Calculi; 29 patients had chronic glomerulonephritis leading to CRF and 3 had reno vascular hypertension. Further, 2 cases with aortic coarctation were found on arteriography; 1 patient had primary aldosteronism; 5 had primary hyperparathyroidism; 6 patients had hyperthyroidism and 11 had Hypothyroidism. In seven cases of hydronephrosis (two known and five newly detected) we could not determine whether the hypertension was caused by the hydronephrosis. The prevalence of curable SH among hypertensive subjects was higher in this screening study, conducted in majority of cases with simple tests.
\end{abstract}

The rates of blood pressure screening in Pakistan are worryingly low; the underlying disease in hypertensive patients should be treated appropriately to avoid long-term use of antihypertensive drugs and risks of atherosclerotic complication. This calls for the establishment of a nationwide program on screening to improve detection, awareness and treatment of hypertension.

Keywords: SH: Secondary Hypertension, CT: Computed Tomography, Angiography, Primary Aldosteronism, Cushing's syndrome, Pheochromocytoma, Renovascular hypertension

\section{Introduction}

The prevalence of hypertension has been well documented in epidemiological studies conducted in many countries. The proportion of patients with curable secondary hypertension, including patients with primary aldosteronism, renovascular hypertension, Cushing's syndrome and pheochromocytoma, to the total hypertensive population has been reported to be from 0.1 to $1.2 \%$ [1-4]. Hypertension is commonin Pakistan, affecting around $18 \%$ of adults aged $>15$ years and $33 \%$ of those $>45$ yearsin urban areas [5-9].

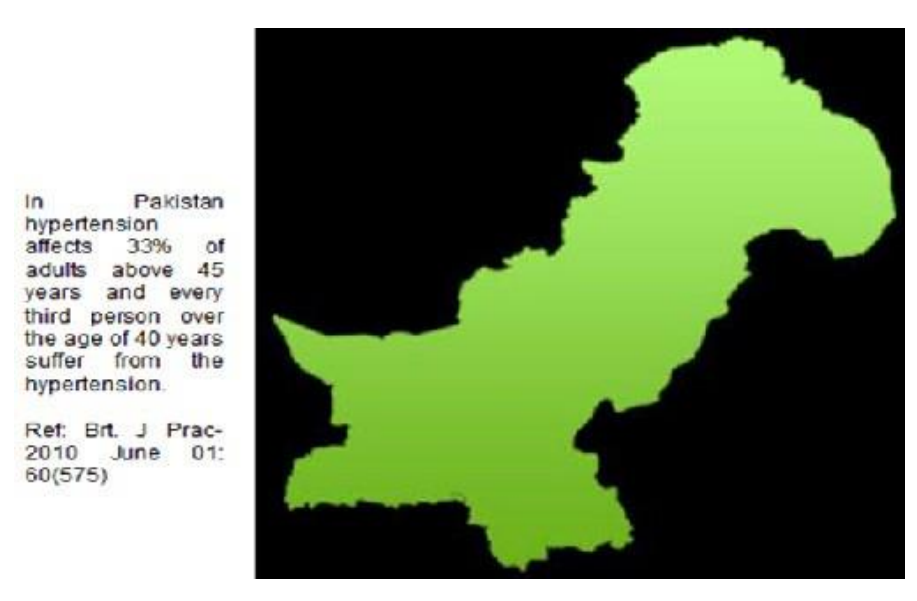

Cardio Open, 2020 
The studies have found good evidence that screening and treatment of high blood pressure in adults substantially reduces the incidence of cardiovascular events. Screening for treatment of overall high blood pressure has few major harms as the net benefit of screening for high blood pressure in adults is substantial, especially when only $5-10 \%$ of patients suffering from increased systemic blood pressure have an identifiable cause [10].

Table 1: Screening of Secondary Hypertension

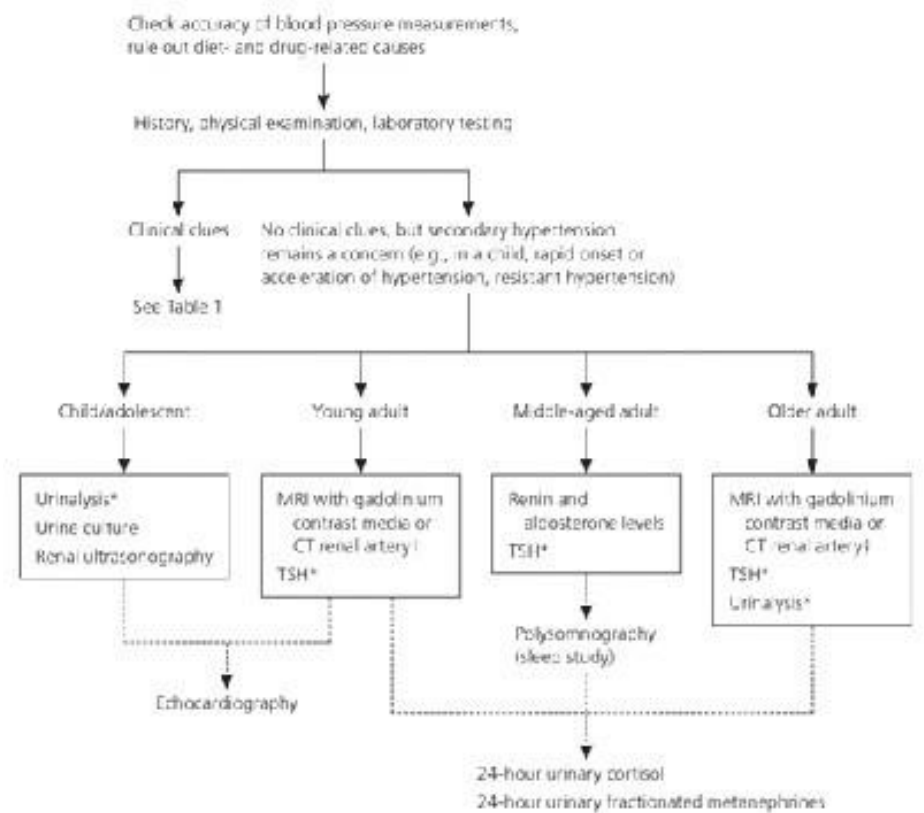

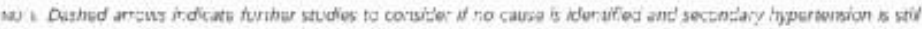
suspoxted.

- If not aone as part at the itico' evalustion

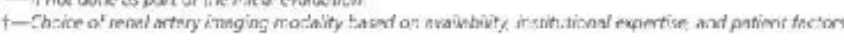

\section{Objectives}

There is a lack of data about hypertension screening in low- to middle-income countries [11] with Pakistan being one of them. The evaluation of a patient with hypertension depends upon the likely cause and the degree of difficulty in achieving acceptable blood pressure control or patients who have clinical clues suggesting the possible presence of secondary hypertension, should undergo a more extensive screening. It is important to be aware of these clues and signs as overall it is not cost effective to perform a complete evaluation for secondary hypertension in every hypertensive patient. Private health clinics account for $70 \%$ of basic health care in Pakistan. The majority of these private clinics are small entities, run by one or more doctors and allied staff.

In general, the care at private clinics does not follow internationally agreed standards of diagnosis and treatment. Thus, the primary objective of this study was to determine the prevalence and predictors of screening for cause of blood pressure in a private clinic in Pakistan. The secondary objective was to identify ways to improve effective BP screening practices among the population at risk from secondary hypertension.

\section{Material and Method}

This study was conducted with prospective analysis of 566 hypertensive patients at Private clinic in Karachi referred by gen- eral practitioners for control of hypertension from May 2017 to June 2018. Medical records of patients $(187 \mathrm{M} / 250 \mathrm{~F})$ were taken, aged $30.1 \pm 8.2$ years (ranging: $15-40$ years), with no family history or other risk factors having maximum systolic blood pressure of $190.8 \pm 29.4 \mathrm{mmHg}$ (ranging: $140-230 \mathrm{~mm} \mathrm{Hg}$ ), and maximum diastolic blood pressure of $108.8 \pm 16.8 \mathrm{mmHg}$ (ranging: $90-150$ $\mathrm{mm} \mathrm{Hg}$ ) which did not improve despite optimal treatment. At least three blood pressure drugs were assessed for an initial screening. 57 patients with essential hypertension were excluded from this study. 437 Hypertensive patients who had been strongly suspected of having Secondary Hypertension were included for further screening. Informed consent was obtained from all subjects after explaining risk. Hypertension was defined as a blood pressure above 140/90 $\mathrm{mmHg}$, according to the classification of BP for adults in the Guidelines for the Management of Hypertension for General Practitioner. These patients under went complete physical examination, and basic laboratory investigations prior to secondary screening $[12,13]$. A secondary aetiology was assessed by symptoms (eg: flushing and sweating suggestive of pheochromocytoma), examination findings (eg: a renal bruit suggestive of renal artery stenosis), or laboratory abnormalities (eg: hypokalaemia suggestive of aldosteronism). Secondary hypertension was also considered in patients with resistant hypertension. Patients under went plasma aldosterone concentration, plasma renin activity, serum cortisol concentration and plasma catecholamine concentration, and their abdominal ultrasonography was performed. They were referred for furosemide plus upright test, captopril renography, dexamethasone suppression test, abdominal ultrasonography (US) abdominal CT and arteriography.

Table 2:Overall Data

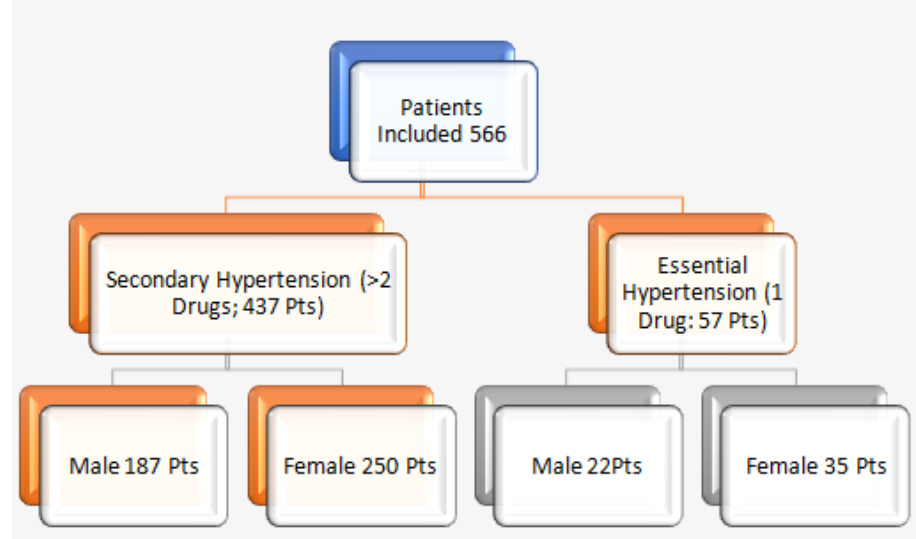

On the basis of the results of these investigations and a thorough examination of each patient's case notes, a diagnosis was recorded using the criteria given below. The diagnosis of chronic glomerulonephritis required persistent or intermittent albuminuria or haematuria, or both without there being another cause of these abnormalities. Plasma catecholamines were measured by ELISA and plasma cortisol by immunochemiluminescent [14]. Orthostatic aldosterone (ELISA) and direct renin (chemiluminescence) were measured in 48 patients. A kidney was considered small if it was over $2 \mathrm{~cm}$ shorter than the other kidney and less than $10 \mathrm{~cm}$ in length with odd shape. Chronic pyelonephritis required a history of recurrent urinary tract infections and a lowered urinary concentration ability. Renovascular Disease Screening was Criteria for Suspected Secondary Hypertension. After Initial Screening 
Patients with a PRA $12.0 \mathrm{ng} / \mathrm{dl}$ were diagnosed as having hyperaldosteronemia, patients with an $\mathrm{F}>20 \mu \mathrm{g} / \mathrm{dl}$ were diagnosed as having hypercortisolemia and they underwent an overnight dexamethasone suppression test. Patients with abnormal US findings of adrenal glands were suspected of adrenal diseases. We used a chi-squared test as the primary statistical test.

Table 3: Blood Pressure Range

\section{BLOOD PRESSURE RANGE}

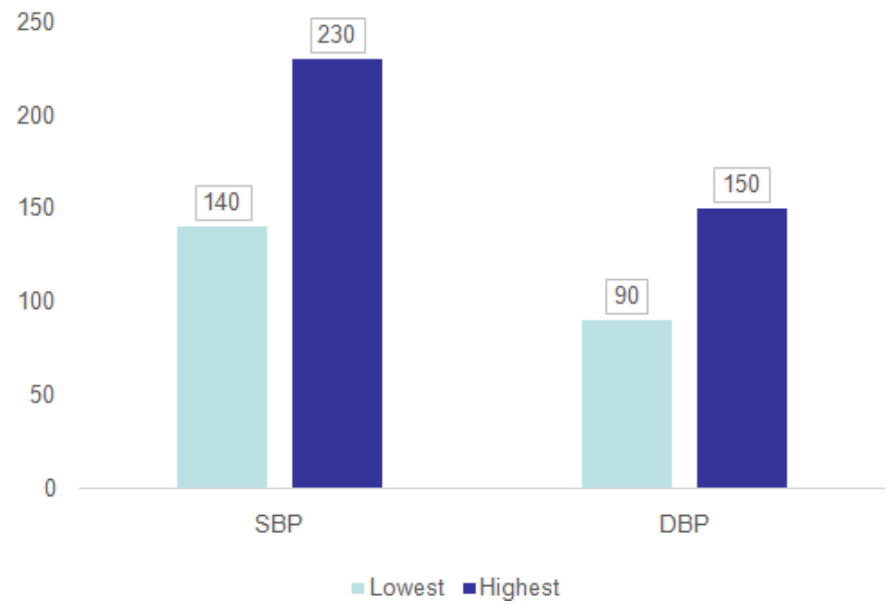

Table 4: Drugs

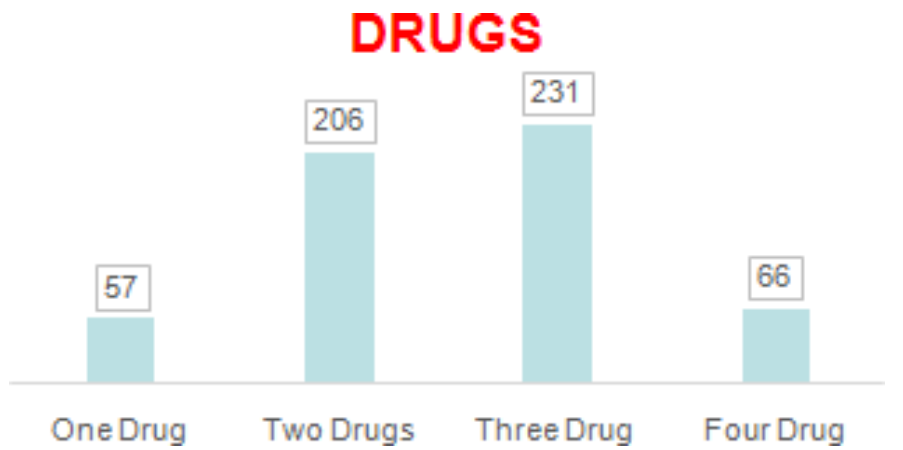

Table 5: ECG

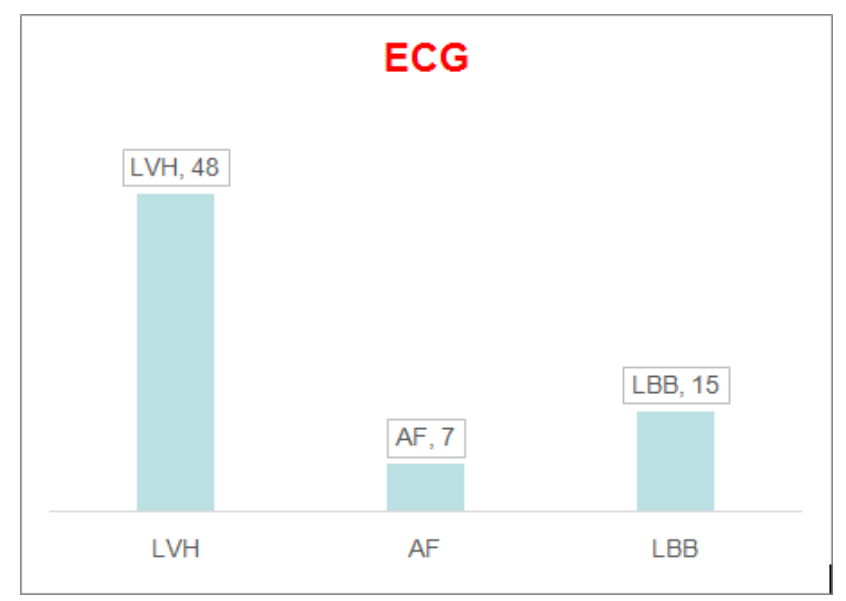

Results

This cross-sectional study was conducted from May 2017 to June 2018. Investigations clearly demonstrated that the prevalence of
Secondary Hypertension, including endocrine and renovascular hypertension, was $8.1 \%$ among 566 hypertensive patients in our clinic. Amongst 437 patients investigated for secondary hypertension, 114 were sent for the Renal pathology, 42 were found to have Renoparenchymal disease; 25 were having Acute Renal Failure. Another 15 patients had Calculi and 29 patients had chronic glomerulonephritis leading to CRF which had been previously confirmed in all cases (by a positive finding on renal biopsy with histopathological examination or proteinuria over $2 \mathrm{~g} /$ day). Persistent haematuria alone was present in three cases test. In seven cases of hydronephrosis (two known and five newly detected), we could not determine whether the hypertension was caused by the hydronephrosis and the resulting partial obstruction to flow. 3 cases had renovascular hypertension with main renal artery stenosis (two uni-lateral and one bilateral) diagnosed by renal arteriography. In addition, there were 2 cases of aortic coarctation on arteriography, one of primary aldosteronism, was clinically diagnosed by adrenocorticotrophic hormone (ACTH)-stimulated adrenal venous sampling, 5 had primary hyperparathyroidism; 6 had hyperthyroidism; 11 patients had hypothyroidism on lab investigations. No case of phaeochromocytoma was discovered. 4 patients with hypertension were cured or improved after, transsphenoidal pituitary adenectomy and 2 patients with coarctation had percutaneous transluminal angioplasty with stenting, all initial and secondary screening tests were found to be sensitive and specific for differentiating SH from essential hypertension (EH).

Table 6: Renal Ethology

\section{RENAL ETIOLOGY}

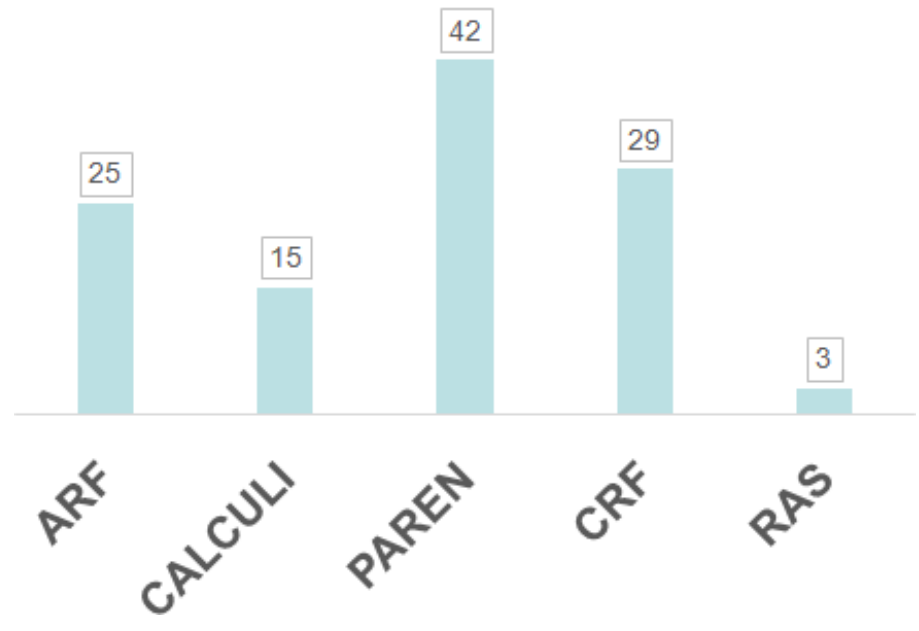

Table 7: Other Ethologies

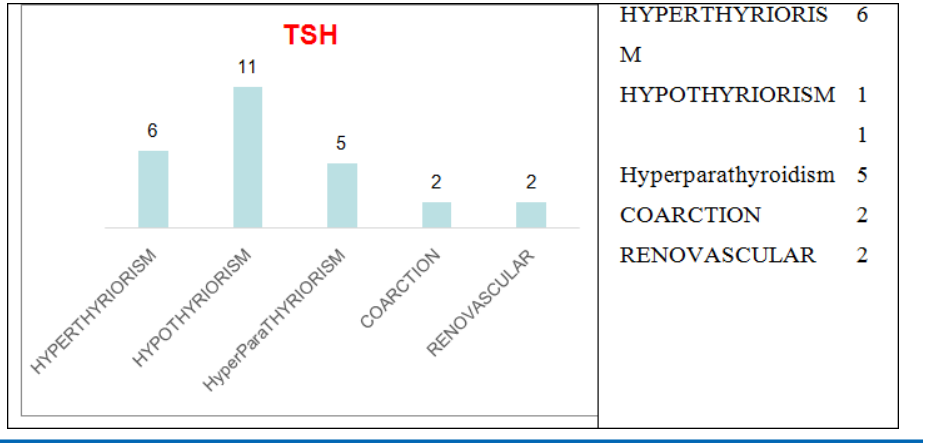

Volume 5 | Issue 3 | 90 


\section{Discussion}

Secondary hypertension is a type of hypertension with an underlying, potentially correctable cause. Our study demonstrated that the prevalence of Secondary Hypertension, including endocrine and renovascular hypertension, was $8.1 \%$ among 566 hypertensive patients in our clinic. Guidelines for the management of hypertension have recommended performing further examinations such as hormonal tests and renography when clinical features are suggestive of Secondary Hypertension [12,13]. In light of some evidence and benefit from a more intense screening program of secondary hypertension, the overwhelming evidence that treating patients diagnosed with secondary hypertension improves patient s' outcomes. But as secondary hypertension is relatively rare, and screening for causes can be expensive and time-consuming, not every patient with high blood pressure will be tested for the underlying condition. Complete screening should be performed only in patients with high clinical suspicion. There are several factors which should be kept in mind when patient is planned for screening of secondary hypertension like identification of some general clinical clues regarding age, habitus, severity of hypertension, history of early onset of hypertension without other risk factors, resistant hypertension on three drugs, or hypertensive emergencies, and sudden increase of BP in previously stable patient. Moreover, it is also important that despite of having found patient appropriately treated for a secondary cause of hypertension, BP rarely ever returns to normal. This indicates either that some patients with secondary hypertension also have concomitant essential hypertension or that vascular remodelling has taken place and progressed over time to the point of no return, so doctors are not following the care at internationally agreed standards of diagnosis and treatment $[12,15]$. The care arrangements at private clinics are not designed to offer long-term care of chronic conditions. Whereas, it is seen that there are some situations that may suggest the presence of a secondary hypertension after starting antihypertensive therapy, a second diagnostic look is indicated if there is an excessive drop in potassium with a small dose of a diuretic for primary aldosteronism or other endogenous or exogenous mineralocorticoid excess. Excessive decrease in GFR with a small dose of an ACE-inhibitor (RAS, predominately when bilateral) in a remarkably resistant arterial hypertension, blood pressure decreases with treatment but remains excessively labile. This study was performed in single centre so we have a limited data and we recommend further such studies in public sector hospitals.

\section{Conclusion}

The present study was performed in a single private clinic of Karachi Pakistan on patients with different social and demographic back ground. The findings on evaluation support the scaling of an integrated secondary hypertension care, as intervention in even urban private clinics are lacking in primary care in Pakistan. Our present prospective study on the prevalence of secondary hypertension among 566 hypertensive patients showed a high prevalence of PA, Cushing's syndrome Hyperthyroidism and Renal disorders. The prevalence of secondary hypertension with curable cases was high [16-18]. Therefore, in patients with a potentially reversible cause of hypertension, early detection and treatment are important to minimize/prevent irreversible changes in the systemic vasculature that may give rise to persistent hypertension with an unfavorable long-term outcome. In comparison, low prevalence of second- ary hypertension, due to surgically curable forms makes routine screening for these cases unnecessary. Our sequential screening system is also not effective method. Therefore, we propose that educating doctors about hypertension and establishing guidelines for diagnosis and care according to our social and economic background and awareness of our data should be our priority and must be taken into account in planning as the first measure in community controlling program for hypertension in Pakistan. Weak regulatory function makes district steered public-private partnership model a possible way to enable and engage the private clinics in delivering better health care and integrated care for hypertension in both rural and urban settings and it should be implemented for treatment of patients with secondary hypertension using appropriate therapies in order to avoid long- term use of antihypertensive drugs for maintaining QOL [8,13,16-19]. Meanwhile we would like to emphasize that all hypertensive patients should be carefully examined for the aetiology of hyper- tension at the first visit to a general outpatient clinic [20].

\section{Reference}

1. Keh-Chuan Loh, Evelyn S Koay, Min-Cheh Khaw, Shanta C Emmanuel, William F Young (2000) Prevalence of primary aldosteronism among Asian hypertensive patients in Singapore. The Journal of Clinical Endocrinology \& Metabolism85: 2854-2859.

2. Haralambos Gavras (2009) Pathogenesis of hypertension: A Review. Journal of Medical Sciences 2: 25-28.

3. Danielson M, Dammström B (1981) The prevalence of secondary and curable hypertension. Acta Med Scand209: 451455.

4. Patricia M Kearney, Megan Whelton, Kristi Reynolds, Paul Muntner, Paul K Whelton, et al. (2005) Global burden of hypertension: analysis of worldwide data. Lancet 365: 217-223.

5. Anderson GH, Blakeman N, Streeten DH (1994) The effect of age on prevalence of secondary forms of hypertension in 4429 consecutively referred patients. J Hypertens 12: 609-615.

6. Jafar TH, Levey AS, Jafary FH, White F, Gul A, et al. (2003) Ethnic subgroup differences in hypertension in Pakistan. J Hypertens 21:905-912.

7. Khabir Ahmad, Tazeen H Jafar (2005) Prevalence and determinants of blood pressure screening in Pakistan. J Hypertens23: 1979-1984.

8. Chataut J, Adhikari RK, Sinha NP (2011) Prevalence and Risk Factors for Hypertension in Adults Living in Central Development Region of Nepal. KUMJ 9: 13-18.

9. Mahesar H, Khand FD, Seehar GM (2011) Prevalence of hypertension and obesity in Hyderabad. Sindh University Research Journal43: 219-224.

10. Tozawa M, Oshiro S, Iseki C, Sesoko S, Higashiuesato Y, et al. (2001) Family history of hypertension and blood pressure in a screened cohort. Hypertens Res24:93-98.

11. Sanjay Basu, Christopher Millett (2013) Social epidemiology of hypertension in middle-income countries: determinants of prevalence, diagnosis, treatment, and control in the WHO SAGE study. Hypertens62:18-26.

12. Giuseppe Mancia, Robert Fagard, Krzysztof Narkiewicz, Josep Redon, Alberto Zanchetti, et al. (2013) ESH/ESC Guidelines for the management of arterial hypertension: The Task Force for the management of arterial hypertension of the Eu- 
ropean Society of Hypertension (ESH) and of the European Society of Cardiology (ESC). Eur Heart J34:2159-2219.

13. Paul AJ, Suzzane O, Barry LC, William CC, Cheryl DH, et al (2014) 2014 Evidence-Based guideline for the management of high blood pressure in adults, Report from the panel members appointed to the Eight Joint National Committee (JNC 8). JAMA311: 507-520.

14. Lynda AS, Christopher BG, Joseph FD, Alpesh A, Frank WP, et al. (2010) Acute kidney injury and cardiovascular outcomes in acute severe hypertension. Circulation 121:2183-2191.

15. Japanese Society of Hypertension Guidelines Subcommittee for the Management of Hypertension (2001) Guidelines for the management of hypertension for general practitioners. Hypertens Res 24: 613-634.

16. Piper MA, Evans CV, Burda BU, Margolis KL, Elizabeth O’Connor, et al. (2014) Screening for High Blood Pressure in
Adults: A Systematic Evidence Review for the U.SPreventive Services Task Force.

17. Albert L Siu, US Preventive Services Task Force (2015) Screening for high blood pressure in adults: U.S. preventive services task force recommendation statement. Ann Intern Med163: 778-786.

18. Shafi ST, Shafi T (2017) A survey of hypertension prevalence, awareness, treatment, and control in health screening camps of rural central Punjab, Pakistan. J Epidemiol Glob Health 7: 135-140.

19. Hussain IM, Naqvi BS, Qasim RM, Ali N (2015) Current trends in treatment of hypertension in Karachi and cost minimization possibilities. Pak J Med Sci 31:1021-1026.

20. Anjum H, Arbab SS, Riffat S (2009) Relation of hypertension with body mass index and age in male and female population of Peshawar, Pakistan. J Ayub Med Coll Abottabad21: 63-65.
Copyright:@2020 Khalida Soomro, This is an open-access article distributed under the terms of the Creative Commons Attribution License, which permits unrestricted use, distribution, and reproduction in any medium, provided the original author and source are credited. 